\title{
Targeting fibrotic integrin
}

\section{By Benjamin Boettner, Associate Editor}

Fibrosis has long been associated with the activation of transforming growth factor- $\beta$ by integrin, but the mechanism underlying the process in vivo was not known. Now, an international team has shown that the myofibroblast-expressed integrin $\alpha_{\mathrm{V}}$ subunit drives fibrogenesis and has proof of concept for therapeutic intervention with a small molecule integrin $\alpha_{\mathrm{V}}$ antagonist from Antegrin Therapeutics LLC. ${ }^{1}$

The biotech is developing an improved version of the lead compound for idiopathic pulmonary fibrosis (IPF).

Fibrosis is a tissue repair process triggered by injury or chronic inflammation that results in excessive accumulation of connective

\section{"The fibrosis models} explored thus far are chemical- or injury-induced models of fibrosis. It will be important to see if the compound works in chronic disease settings and in models of fibrosis where chronic infection like $\mathrm{HCV}$ is a major driver of fibrosis."

-Thomas Wynn,

National Institute of Allergy and Infectious Diseases tissue, which over time can lead to permanent scarring and organ failure. Fibrosis occurs in endstage liver disease, IPF and kidney disease. $^{2}$

In the liver, myofibroblasts produce integrin $\alpha_{\mathrm{V}}$ (CD51)containing complexes on their surface that activate latent transforming growth factor- $\beta$ (TGFB; TGF $\beta$ ) reservoirs in the extracellular matrix of different cell types. TGFB in turn induces myofibroblasts to produce collagen and other extracellular matrix proteins that constitute the fibrotic tissue.

At the molecular level, in vitro and in vivo studies have shown that TGFB activation results from integrin $\alpha_{\mathrm{V}}$ subunit-containing complexes interacting with an arginine-glycine-aspartic acid (RGD) motif on TGFB. Whether this mechanism also occurs in myofibroblasts and actually drives fibrosis in vivo had yet to be determined. A key impediment was the lack of a genetic system for manipulating myofibroblasts in vivo.

An international team led by Dean Sheppard, a professor in the Department of Medicine and director of the Lung Biology Center at the University of California, San Francisco, has now devised a strategy to genetically manipulate liver-specific myofibroblasts in mice.

The researchers controlled gene activation in myofibroblasts by generating mice with conditional knockout of pericytes, a class of cells that envelop and protect capillaries and venules in a variety of tissues and organs. ${ }^{3}$ The team targeted hepatic stellate cells (HSCs) - the liver-specific pericytes and direct precursors of myofibroblasts in liverto specifically manipulate gene expression in myofibroblasts.

In mice lacking myofibroblast integrin $\alpha_{\mathrm{V}}$ expression, attempts to induce fibrosis led to low levels of fibrosis markers such as actin $\alpha 2$ smooth aorta muscle (Acta2; $\alpha$-Sma) compared with levels seen in mice expressing the integrin $\alpha_{\mathrm{V}}$ subunit. The mice lacking integrin $\alpha_{\mathrm{v}}$ expression also developed minimal fibrosis.

In culture, HSCs from the mice lacking integrin $\alpha_{\mathrm{V}}$ expression also produced substantially less active TGFB1 and had decreased TGFB1 signaling compared with HSCs expressing the integrin $\alpha_{\mathrm{V}}$ subunit.

A similar mechanism was at work in the lung and kidney, in which myofibroblasts also drive fibrosis. Indeed, disrupting integrin $\alpha_{v}$ in an equivalent cell population in these tissues protected mice from developing fibrosis.

Finally, the researchers provided evidence that targeting profibrotic integrin $\alpha_{\mathrm{v}}$ with a small molecule RGD peptidomimetic antagonist, CWHM 12, inhibited liver and pulmonary fibrosis progression in mice. CWHM 12 competes with the activating RGD motif in latent TGFB1 for integrin $\alpha_{\mathrm{V}}$ binding.

Results were published in Nature Medicine.

The team included researchers from The University of Edinburgh, Saint Louis University, The University of Texas MD Anderson Cancer Center, Harvard Medical School, Uppsala University, the Karolinska Institute, the Max Planck Institute for Molecular Biomedicine and the University of Muenster.

UCSF has filed for a patent covering the identification of integrin $\alpha_{\mathrm{v}}$ complexes.

"Integrin $\alpha_{\mathrm{v}}$ inhibition on myofibroblasts as the theme emerging from the study builds on a growing body around mechanisms underlying fibrosis, including pathways that are conserved between organs," said Scott Friedman, dean for therapeutic discovery and a professor of medicine, liver diseases, pharmacology and systems therapeutics at the Icahn School of Medicine at Mount Sinai. "The treatment strategy is a nice example showing that antagonizing a conserved pathway could impact fibrosis in more than one organ. Integrin $\alpha_{\mathrm{V}}$ is eminently targetable and accessible to inhibitory compounds, including antibodies."

Thomas Wynn, chief of the immunopathogenesis section of the NIH's National Institute of Allergy and Infectious Diseases, agreed. "This is indeed a very exciting study as it suggests integrin $\alpha_{v}$ inhibition in myofibroblasts may prove highly efficacious in a wide variety of fibrotic diseases," he said.

\section{The myofibroblast advantage}

According to Sheppard, one of the major advantages of his team's strategy is that targeting integrin-mediated TGFB1 activation has the potential advantage of decreasing toxicity compared with targeting all TGFB1.

"This could be an important opportunity, since no drug specifically tackling fibrosis in any organ is available," he said.

Scott Turner, VP of R\&D at biomarker discovery company KineMed Inc., said that it will be important to determine the effect of inhibiting integrin $\alpha_{\mathrm{V}}$ in healthy tissues. "This will inform about safety liabilities. 
In addition, there could be a chance that the integrin might play a role in normal wound healing and scarring processes apart from fibrosis," he said.

According to Friedman, broadly targeting TGFB1 directly could have adverse effects such as infection or neoplasia. "The appeal of the present study is that it circumvents this risk by rendering TGFB1 inhibition cell type and tissue specific, thus minimizing the risk," he said.

Wynn said that it will be important to determine whether targeting the integrin $\alpha_{\mathrm{v}}$ subunit with a small molecule inhibitor will prove safe and effective in the long term "given that the TGFB1 pathway is potentially dangerous to disrupt over a long period of time. It also will be vital to find out if the small molecule inhibitor only works by blocking the TGFB1 pathway or if it also works by blocking other profibrotic mechanisms."

Wynn also noted that TGFB1 is not the only mediator of fibrosis.

Sheppard told SciBX that his team is planning to determine how integrin-mediated TGFB1 activation contributes to different fibrotic processes. He also said that the team is focusing on identifying the integrin $\beta$ subunits in complex with integrin $\alpha_{\mathrm{V}}$ that are relevant to fibrosis.

"The fibrosis models explored thus far are chemical- or injuryinduced models of fibrosis," noted Wynn. "It will be important to see if the compound works in chronic disease settings and in models of fibrosis where chronic infection like $\mathrm{HCV}$ is a major driver of fibrosis. Long-term toxicity studies will be needed before the drug is used in humans, and it will also be important to determine if it is efficacious in models other than mice".

"Each fibrosis indication requires its own set of preclinical models. For small molecules like the one featured in the study, the therapeutic window is always a critical parameter to assess," noted Shelia Violette, senior director of the tissue injury and fibrosis research unit at Biogen Idec Inc. "In addition, developing a biomarker strategy that can help read out blockade of the target in early clinical trials can be of great use. These readouts should provide proof of biology and a sense of specificity and saturation of the integrin $\alpha_{\mathrm{V}}$ target by the compound."

Biogen's STX-100, a mAb specific to integrin $\alpha_{v} \beta_{6}$ complexes, is in Phase II testing to treat IPF.

\section{Enter Antegrin}

Antegrin, a spinout of the Center for World Health \& Medicine at Saint Louis University, is developing therapies for fibrotic diseases with an initial focus on IPF. The company provided the integrin $\alpha_{\mathrm{V}}$ inhibitor CWHM 12 for the published study. David Griggs and Peter Ruminski, cofounders and scientific advisers at the company, are coauthors of the paper.

Griggs is director of cellular and molecular biology at the Center for World Health \& Medicine and an adjunct assistant professor of pharmacology and physiology at the Saint Louis University School of Medicine. Ruminski is the center's executive director.

Saint Louis University has filed for patents covering composition of matter and use of integrin inhibitors. Antegrin has an option to obtain an exclusive license to the IP.

Griggs told SciBX that "although CWHM 12 has been an excellent tool compound for early studies, later unpublished compounds are superior in regard to aqueous solubility and potency to inhibit integrin $\alpha_{\mathrm{v}}$. Antegrin is synthesizing and profiling additional integrin inhibitors from which to select the candidate(s) for further development."

Ruminski added, “Antegrin's next big milestone is the selection of a clinical development candidate for inhalation delivery, which we think will be attractive for treatment of idiopathic pulmonary fibrosis. Our compounds have physicochemical properties that make them favorable for pulmonary delivery."

This delivery method can reach higher concentrations with less toxicity in the lung, according to the company.

Boettner, B. SciBX 6(46); doi:10.1038/scibx.2013.1308

Published online Dec. 5, 2013

\section{REFERENCES}

1. Henderson, N.C. et al. Nat. Med.; published online Nov. 10, 2013; doi: $10.1038 / \mathrm{nm} .3282$

Contact: Dean Sheppard, University of California, San Francisco, Calif.

e-mail: dean.sheppard@ucsf.edu

Contact: Neil C. Henderson, The University of Edinburgh,

Edinburgh, U.K.

e-mail: neil.henderson@ed.ac.uk

2. Wynn, T.A. \& Ramalingam, T.R. Nat. Med. 18, 1028-1040 (2012)

3. Foo, S.S. et al. Cell 124, 161-173 (2006)

COMPANIES AND INSTITUTIONS MENTIONED

Antegrin Therapeutics LLC, St. Louis, Mo.

Biogen Idec Inc. (NASDAQ:BIIB), Weston, Mass.

Center for World Health \& Medicine at Saint Louis University,

St. Louis, Mo.

Harvard Medical School, Boston, Mass.

Icahn School of Medicine at Mount Sinai, New York, N.Y.

Karolinska Institute, Stockholm, Sweden

KineMed Inc., Emeryville, Calif.

Max Planck Institute for Molecular Biomedicine,

Muenster, Germany

National Institute of Allergy and Infectious Diseases,

Bethesda, Md.

National Institutes of Health, Bethesda, Md.

Saint Louis University, St. Louis, Mo.

Saint Louis University School of Medicine, St. Louis, Mo.

University of California, San Francisco, Calif.

The University of Edinburgh, Edinburgh, U.K.

University of Muenster, Muenster, Germany

The University of Texas MD Anderson Cancer Center,

Houston, Texas

Uppsala University, Uppsala, Sweden 